\title{
Long-Term Recurrent Hypoxia in Developing Rat Attenuates Respiratory Responses to Subsequent Acute Hypoxia
}

\author{
IMMANUELA RAVÉ MOSS, MÉLANIE BÉLISLE, AND ANDRÉ LAFERRIÈRE \\ Department of Pediatrics [I.R.M., M.B., A.L.], Department of Physiology [I.R.M.], McGill University, Montreal Children's Hospital \\ Research Institute, Developmental Respiratory Laboratory, Montreal, Quebec, Canada H3H 1 P3
}

\begin{abstract}
Whereas definitive treatment of pediatric conditions associated with hypoxemia reverses many pathologic symptoms, some physiologic dysfunctions appear to persist. These abnormalities are attributed to long-lasting central effects of prior hypoxia. To investigate such effects in an animal model, male rats were exposed to $\mathrm{Fio}_{2}=0.12$ continuously for $7 \mathrm{~h}$ daily from postnatal day (p) 17 (representing early childhood) through p33 (representing adolescence), defined as recurrent hypoxia. Respiratory responses during and following 20 min $\mathrm{FiO}_{2}=0.12$ were measured on p35 and p47. To control for early weaning on p15 (normal weaning $=$ p21), male rats were weaned on either $\mathrm{p} 15$ or $\mathrm{p} 21$, raised in normoxia, and also tested for respiratory responsiveness to acute hypoxia. To assess sex differences, female rats were assigned to similar groups and protocols. Minute ventilation, respiratory frequency, tidal volume, and respiratory drive were measured in unsedated animals using whole-body plethysmography. After recurrent hypoxia, male rats displayed an attenuation of ventilation, frequency, and drive during hypoxia, and of all functions after hypoxia on both p35 and p47. There were no differences between test days during hypoxia, and greater attenuation of tidal volume and respiratory drive on p47 during recovery from hypoxia. Respiratory responses displayed no effect of sex on p35, and occasional effects of early weaning on p35 and p47. Thus, recurrent hypoxia produces long-lasting attenuation in respiratory responsiveness to subsequent acute hypoxia. Such long-lasting attenuation, if present in humans, may diminish the protection of children with a history of recurrent hypoxemia against future hypoxic events. (Pediatr Res 59: 525-530, 2006)
\end{abstract}

$\mathrm{H}$ ypoxemia of varying duration, pattern, and timing is an important feature in many clinical conditions in pediatrics. A strong association or causality between hypoxemia and developmental, behavioral, and/or cognitive dysfunctions has been documented, primarily for sleep-disordered breathing and congenital heart disease (1). With regard to sleepdisordered breathing, the dysfunctions also include sleep fragmentation, diminished arousability, diminished responsiveness to respiratory chemical stimuli, fatigue, failure to thrive, hypertension, and cor pulmonale (2-6).

Received February 28, 2005; accepted November 11, 2005

Correspondence: Immanuela Ravé Moss, M.D., Ph.D., Departments of Pediatrics and Physiology, McGill University, The Montreal Children's Hospital, Room A-707, 2300 Tupper St., Montreal, Quebec H3H 1P3, Canada; e-mail: Immanuela. Moss@muhc.mcgill.ca

This work was supported by the Canadian Institutes of Health Research and the Montreal Children's Hospital Research Institute (I.R.M.) and by a McGill University Research Bursary to Medical Students (M.B.).

DOI: 10.1203/01.pdr.0000203104.45807.23
Whereas adenotonsillectomy, a definitive treatment for sleep-disordered breathing associated with enlarged adenoids and/or tonsils, reverses the upper airway obstruction and many sleep-related symptoms in children, recent studies suggest that abnormalities in cardiorespiratory control, arousability, learning, and behavior can persist beyond the relief of such obstruction (4,7-9). These studies have attributed the lingering dysfunctions to long-term central effects of prior hypoxemia associated with more severe obstructive sleep apnea.

It is important to establish in controlled animal studies whether there indeed are long-lasting effects of an antecedent hypoxia. It is also important to determine whether such effects, if present, are particular to a specific pattern of prior hypoxia: Do they only follow a recurrent intermittent hypoxia that is characteristic of obstructive sleep apnea, or do they also follow a continuous hypoxia or recurrent prolonged episodes of hypoxia that are more common to entities such as congenital heart disease or protracted lung disease?

Long-term effects of postnatal hypoxia on subsequent respiratory control have been investigated. Rat pups exposed to continuous hypoxia from postnatal day 1 (p1) to $\mathrm{p} 6$ or $\mathrm{p} 7$ have been reported to display attenuated respiratory responses to acute hypoxia $7-10(10)$ or $12 \mathrm{wk}$ later $(11,12)$. These findings are important in that they indicate in the rat model that continuous hypoxia in the neonatal period can cause persistent respiratory control abnormalities in adulthood. However, the age of hypoxic exposure in these studies is considered equivalent to human prematurity in terms of brain development (13), and does not match the age range during which obstructive sleep apnea and many lung or congenital heart diseases prevail $(1,2,14)$. It is thus unknown whether continuous or discontinuous hypoxia during more advanced postnatal development in rat is also associated with subsequent respiratory control abnormalities.

The current study examined whether recurrent hypoxia throughout a period equivalent to human childhood in rat would also elicit an attenuation in respiratory responsiveness to subsequent acute hypoxia. Accordingly, hypoxic exposure $\left(\mathrm{FiO}_{2}=0.12\right.$ for $\left.7 \mathrm{~h}\right)$ was started on p17, an age associated with maturing stress responses $(15,16)$ and respiratory responsiveness to hypoxia (17), thus representing early childhood in rat. This hypoxic pattern was repeated on 17 consecutive days (thus termed recurrent hypoxia), and was followed by assessment of respiratory responsiveness to acute hypoxic exposure at two time intervals (p35 and p47). 
The recurrent hypoxia protocol required early weaning (normal weaning on p21) and maternal separation of the rat pups. Early separation is a stressor that carries long-term consequences, especially when it occurs between p3 and p14 in rat, as this period, known as the hyporesponsive period to stress, is associated with an immature hypothalamic-pituitaryadrenal axis $(15,16)$. Exposure to any stressor, including hypoxia, during that time interval leads to long-term, sexspecific perturbations in many functions $(15,16)$, including subsequent responsiveness to acute hypoxia (18). Whereas the studies of continuous neonatal hypoxia cited above (10-12) avoided maternal separation by exposing both the pups and their dams to hypoxia together, the hypoxic exposure occurred during the hyporesponsive stress period and was compounded by growth retardation in the exposed pups. To avoid subjecting pups to the major stresses associated with early weaning, the rat pups were weaned on $\mathrm{p} 15$ and provided with nutritional supplement, group housing, and daily handling. Additional rat groups, weaned on either p15 or p21 and raised in normoxia, were subjected to the same acute hypoxia protocols, thus serving as controls for the stress effects of maternal separation and recurrent hypoxia.

The test days, p35 and p47, on which respiratory responsiveness to acute hypoxia was measured, are postnatal ages at which estrous cycles might begin to affect breathing. Differences between males and females in responsiveness to hypoxia have indeed been reported (10). Whereas this study focused on responsiveness in male rats, female rat groups were assigned to the same recurrent hypoxia and control study groups, and their responsiveness to a subsequent acute hypoxia test was compared on p35 to control for the effects of sex on respiratory control at that time.

\section{MATERIALS AND METHODS}

General experimental design. The main experiment assessing whether antecedent hypoxia resulted in long-term respiratory control abnormalities was conducted in male rats. Male pups were weaned early, on p15, exposed to recurrent hypoxia from p17 through $\mathrm{p} 33$ (P15RH group), and then tested for respiratory responsiveness to an acute hypoxic exposure on $\mathrm{p} 35$ or $\mathrm{p} 47$. Control groups comprised male pups weaned early on p15, or normally on $\mathrm{p} 21$, subjected to normoxia ( $\mathrm{P} 15 \mathrm{~N}$ and $\mathrm{P} 21 \mathrm{~N}$, respectively), and also tested for respiratory responsiveness to an acute hypoxic exposure on p35 or p47.

To control for sex effects, female pups were assigned to the same recurrent hypoxia and normoxia groups and protocols, and were tested for respiratory responsiveness to an acute hypoxic exposure on $\mathrm{p} 35$. Because there were no differences in respiratory responsiveness to acute hypoxia between females and males on $\mathrm{p} 35$, no females were studied beyond that postnatal age. To evaluate general stress effects of early weaning and hypoxia on growth, pups of both sexes and all study groups were weighed every $1-6 \mathrm{~d}$ from p15 up to p35.

Animals, weaning, nutrition. Pregnant Sprague-Dawley females (Charles River Canada, St. Constant, QC, Canada) were obtained 3-5 d before parturition. Each litter was kept with its dam in a standard rodent cage under constant temperature, humidity, and a 12-h (0700-1900 h) light/dark cycle. On p13, all pups were introduced in the maternal cage to a wet mash consisting of crushed standard rat food (Purina) mixed 1:1 (vol) with sweetened and condensed milk (Borden's) diluted 1:1 in water. Each pup was also handled for 10 min daily. On p15, randomly selected male and female rat pups were separated from their dam and housed in litter-specific groups. Weaned pups were then handled daily and fed sweetened mash in addition to standard rodent food and water ad libitum until the normal weaning day, p21. Pups not weaned early remained with their dam, were weaned on p21, and then were housed in litter-specific groups. From p21 onwards, all rats were handled daily and given standard rodent food.

All animals were cared for in accordance with the Canadian Guide for the Care and Use of Laboratory Animals, and their use was approved by the institutional review board.
Experimental protocols. Pups weaned on p15 and assigned to the recurrent hypoxia protocol (P15RH), or those weaned on p15 or p21 and assigned to the normoxia protocol (P15N and $\mathrm{P} 21 \mathrm{~N}$, respectively), were placed in groups of four to six for $7 \mathrm{~h}$ daily $(0900-1600 \mathrm{~h}$, during the sleep phase for rat) in a sealed acrylic box $(41 \mathrm{~cm} \mathrm{H} \times 22 \mathrm{~cm} \mathrm{~W} \times 41 \mathrm{~cm} \mathrm{~L})$ through which either $\mathrm{FiO}_{2}=0.12 \pm 0.01$ or a normoxic mixture $\left(\mathrm{FiO}_{2}=0.21\right)$ was flowed at 10 $\mathrm{L} / \mathrm{min}$. Gases were monitored continuously by $\mathrm{O}_{2}$ and $\mathrm{CO}_{2}$ analyzers (Biopac Systems, Santa Barbara, CA). The concentration of $\mathrm{CO}_{2}$ in the box was $0.3-0.6 \%$. Two small holes in the box prevented a buildup of air pressure. Food and water were freely available in the box during the 7-h period. The box was maintained at $24^{\circ} \mathrm{C}$ and was fitted with a raised, slatted floor under which moisture-absorbent material was placed. After each 7-h period, rat pups were returned to their home cages. This exposure was repeated daily from p17 through $\mathrm{p} 33$. For the hypoxia protocol, this repeated exposure was termed recurrent hypoxia.

On p33 and p34, to familiarize the rats with the respiratory testing environment, each pup was placed for $10 \mathrm{~min}$ in a flow-through whole-body plethysmograph (Buxco Electronics, Sharon, CT), through which regulated gases were flowed at $2.0 \mathrm{~L} / \mathrm{min}$. On p35 (males and females), or on p47 (males), tidal volume, respiratory frequency, minute ventilation, and respiratory drive (tidal volume/inspiratory time) were measured in unrestrained rats in the plethysmograph. Oxygen and $\mathrm{CO}_{2}$ concentrations, humidity, and temperature within the chamber were measured with sensors (Buxco), and body temperature was measured with a rectal probe immediately before the recording session (Cole Parmer, Model YSL-55, Vernon Hills, IL). After an initial period of habituation to the chamber, respiratory measurements were recorded for 6 min in $\mathrm{FiO}_{2}=0.21$ (normoxia) while the rat was awake and quiet (baseline), then for $20 \mathrm{~min}$ in $\mathrm{Fio}_{2}=0.12$ (hypoxia), then again for 20 min in $\mathrm{FiO}_{2}=0.21$ (recovery in normoxia).

Data analysis and statistics. Respiratory measures, corrected for body temperature, barometric pressure, and relative humidity (19), were averaged every 2 min during the baseline, hypoxia, and recovery periods of the recording session. This yielded three baseline measures, the median of which was used as the baseline value for that session, 10 consecutive periods in hypoxia, and 10 consecutive periods during recovery in normoxia. The respiratory variables (tidal volume, respiratory frequency, minute ventilation, and respiratory drive) measured during each sampling period were averaged by group (P15RH, P15N, P21N) and sex.

Raw measures of respiratory indices and temperature at baseline were compared among the groups by a one-way ANOVA.

Respiratory responsiveness during acute hypoxia and, separately, during the recovery from acute hypoxia in normoxia was calculated from the difference between the respiratory measure at each sampling period and the baseline for each animal. This difference was also divided by the same baseline value and multiplied by 100 to obtain a measure of change in respiration for that animal expressed as a percent of its baseline. These measurements were sorted by study group and averaged. Normality of group means at each time segment of the hypoxic or recovery periods was assessed by Levene test. Group means were then analyzed by a repeated-measures ANOVA, the factors of which were study group, test day, and time during acute hypoxia or during recovery from hypoxia. Significant main effects or interactions were followed by post hoc pairwise Duncan multiple range tests to identify differences between groups. Using Dunnett's test, additional post hoc pairwise comparisons of raw ventilatory measures during recovery from acute hypoxia were used to estimate the time it took these measures to return to baseline. Differences between sexes were also analyzed using a repeatedmeasures ANOVA applied to the data obtained on $\mathrm{p} 35$, the factors of which were sex, study group, and time in hypoxia or time in recovery from hypoxia.

In addition, absolute changes in minute ventilation above baseline on p35 and $\mathrm{p} 47$ were averaged over the period of acute hypoxia or recovery from hypoxia and compared among male groups using a two-way ANOVA, the factors of which were study group and test day. Significant effects were followed by post hoc pairwise Duncan multiple range tests to identify differences between groups.

Body weights collected at 1- to 6-d intervals were grouped by study group and sex, and analyzed by a repeated-measures ANOVA, the factors of which were study groups, sex, and postnatal days. Significant effects and interactions were followed by post hoc pairwise Duncan tests to identify time points at which group differences existed.

Significance was determined at $p \leq 0.05$ throughout (Statistica, version 7, StatSoft, Tulsa, OK).

\section{RESULTS}

Animals. Results were obtained from 76 rats: 1) male P15RH rats tested in acute hypoxia on $\mathrm{p} 35(n=6)$ or $\mathrm{p} 47$ $(n=8) ; 2)$ male $\mathrm{P} 15 \mathrm{~N}$ rats tested on $\mathrm{p} 35(n=11)$ or $\mathrm{p} 47$ 
$(n=9) ; 3)$ male $\mathrm{P} 21 \mathrm{~N}$ rats tested on $\mathrm{p} 35(n=7)$ or $\mathrm{p} 47(n=$ $8)$; 4) female P15RH rats tested on p35 $(n=6)$; 5) female $\mathrm{P} 15 \mathrm{~N}$ rats tested on $\mathrm{p} 35(n=11)$; and 6$)$ female $\mathrm{P} 21 \mathrm{~N}$ rats tested on p35 $(n=10)$.

Effect of recurrent hypoxia on respiratory responses to subsequent acute hypoxia in male rats. On p35 and p47, group differences at baseline normoxia (Table 1) were found among the study groups in minute ventilation $(\mathrm{F}(2,43)=$ $4.675, p=0.014)$, respiratory frequency $(\mathrm{F}(2,43)=5.428$, $p=0.008)$ and respiratory drive $(\mathrm{F}(2,43)=7.142, p=$ $0.002)$. These differences resulted from higher levels of these variables in the $\mathrm{P} 15 \mathrm{RH}$ group than in the $\mathrm{P} 15 \mathrm{~N}$ group $(p<$ $0.05)$. In addition, the $\mathrm{P} 15 \mathrm{RH}$ group displayed a higher minute ventilation than the $\mathrm{P} 21 \mathrm{~N}$ group on $\mathrm{p} 35(p<0.05)$, and a higher respiratory drive than the $\mathrm{P} 21 \mathrm{~N}$ group on $\mathrm{p} 47$ ( $p<$ $0.05)$. No difference in any respiratory parameter was found between test days.

During acute hypoxia on both p35 and p47 (Fig. 1), all respiratory functions in each study group displayed an initial rapid rise to a peak value 4-8 min after the onset of hypoxia, and, thereafter, a gradual and variable decline over the remaining hypoxic period. Therefore, irrespective of antecedent hypoxia or weaning history, there was a significant change over time spent in acute hypoxia in minute ventilation $(\mathrm{F}(9,387)=7.364, p<0.0001)$, respiratory frequency $(\mathrm{F}(3,387)=2.311, p=0.002)$, tidal volume $(\mathrm{F}(9,387)=$ $8.562, p<0.0001)$, and respiratory drive $(\mathrm{F}(3,387)=6.966$, $p<0.0001$ ), each expressed as mean percentage change from baseline. The magnitude of responsiveness during acute hypoxia, however, differed among study groups on both p35 and p47, with no change between test days (Fig. 1). Group differences occurred in minute ventilation $(\mathrm{F}(2,43)=8.123, p=$ $0.001)$, respiratory frequency $(\mathrm{F}(2,43)=9.045, p=0.006)$, and respiratory drive $(\mathrm{F}(2,43)=12.002, p<0.0001)$.
$\mathrm{P} 15 \mathrm{RH}$ rats displayed a smaller increase in minute ventilation during acute hypoxia than $\mathrm{P} 15 \mathrm{~N}(p=0.0005)$ or $\mathrm{P} 21 \mathrm{~N}$ rats $(p=0.0456)$. The difference between the $\mathrm{P} 15 \mathrm{RH}$ and the $\mathrm{P} 15 \mathrm{~N}$ groups occurred throughout the hypoxic challenge (pairwise comparisons, Fig. 1). The effect of recurrent hypoxia on the P15RH group was seen not only as a smaller percentage increase in minute ventilation (Fig. 1), but also as a significantly smaller absolute ventilatory response to hypoxia averaged over the entire hypoxia test period $(\mathrm{F}(2,43)=8.123, p=$ 0.0011 ) (Fig. 2A). On p35, P15N rats also displayed a higher average minute ventilation during hypoxia than did $\mathrm{P} 21 \mathrm{~N}$ rats (Fig. 2A).

Similarly, on p35 and p47, P15RH pups displayed a smaller frequency increase than did P15N animals $(p=0.0001)$ throughout the acute hypoxia (pairwise comparisons, Fig. 1). $\mathrm{P} 15 \mathrm{~N}$ rats displayed higher frequency responses to hypoxia than did $\mathrm{P} 21 \mathrm{~N}$ rats ( $p=0.002$; pairwise comparisons, Fig. 1). Average inspiratory time response on 477 decreased less in $\mathrm{P} 15 \mathrm{RH}$ than in $\mathrm{P} 15 \mathrm{~N}$ or $\mathrm{P} 21 \mathrm{~N}$ groups $(p=0.0015, p=$ 0.0125 , respectively), whereas average expiratory time response decreased to the same extent in all groups, thus mirroring the changes in respiratory frequency (data not shown).

Whereas there were no group differences in tidal volume (Fig. 1), P15RH rats displayed a smaller increase in respiratory drive $\left(\mathrm{V}_{\mathrm{T}} / \mathrm{T}_{\mathrm{I}}\right)$ than did either P15N $(p=0.0001)$ or $\mathrm{P} 21 \mathrm{~N}$ rats $(p=0.0022)$, these differences occurring during most of the hypoxic period (pairwise comparisons, Fig. 1).

Effect of recurrent hypoxia on respiration during recovery from acute hypoxia in male rats. Overall, upon cessation of hypoxia, each respiratory parameter decreased rapidly, often below baseline, then changed gradually toward prehypoxic baseline (Fig. 1), thus displaying a change over time regardless of hypoxic history or weaning conditions (minute ventilation: $\mathrm{F}(9,387)=5.427, p<0.0001$; respiratory frequency:

Table 1. Effects of age at weaning and group assignment on baseline respiratory indices and rectal temperature in male rats.

\begin{tabular}{|c|c|c|c|}
\hline \multirow[b]{2}{*}{ Measures } & \multicolumn{3}{|c|}{ Test day p35 [males $(N=24)]$} \\
\hline & $\mathrm{P} 21 \mathrm{~N}(n=7)$ & $\mathrm{P} 15 \mathrm{~N}(n=11)$ & $\mathrm{P} 15 \mathrm{RH}(n=6)$ \\
\hline $\mathrm{TR}\left({ }^{\circ} \mathrm{C}\right)$ & $36.7 \pm 0.2$ & $36.9 \pm 0.1$ & $36.6 \pm 0.3$ \\
\hline$\dot{\mathrm{V}}(\mathrm{mL} / \mathrm{min} / 100 \mathrm{~g})$ & $103.1 \pm 8.1$ & $96.0 \pm 6.5$ & $128.4 \pm 8.7 * \dagger$ \\
\hline $\mathrm{f}_{\mathrm{R}}$ (breaths/min) & $127.7 \pm 7.0$ & $122.5 \pm 4.6$ & $129.2 \pm 7.6^{*}$ \\
\hline $\mathrm{V}_{\mathrm{T}}(\mathrm{mL} / 100 \mathrm{~g})$ & $0.80 \pm 0.07$ & $0.84 \pm 0.06$ & $0.99 \pm 0.04$ \\
\hline \multirow[t]{2}{*}{$\mathrm{V}_{\mathrm{T}} / \mathrm{T}_{\mathrm{I}}(\mathrm{mL} / 100 \mathrm{~g} / \mathrm{s})$} & $4.30 \pm 0.32$ & $4.23 \pm 0.22$ & $5.04 \pm 0.11 *$ \\
\hline & \multicolumn{3}{|c|}{ Test day p47 [males $(N=25)]$} \\
\hline Measures & $\mathrm{P} 21 \mathrm{~N}(n=8)$ & $\mathrm{P} 15 \mathrm{~N}(n=9)$ & $\mathrm{P} 15 \mathrm{RH}(n=8)$ \\
\hline $\mathrm{TR}\left({ }^{\circ} \mathrm{C}\right)$ & $36.5 \pm 0.2$ & $36.4 \pm 0.2$ & $36.2 \pm 0.2$ \\
\hline$\dot{\mathrm{V}}(\mathrm{mL} / \mathrm{min} / 100 \mathrm{~g})$ & $98.3 \pm 7.6$ & $93.2 \pm 7.1$ & $106.2 \pm 7.6^{*}$ \\
\hline $\mathrm{f}_{\mathrm{R}}($ breaths/min$)$ & $127.0 \pm 4.7$ & $119.3 \pm 4.2$ & $134.8 \pm 5.1^{*}$ \\
\hline $\mathrm{V}_{\mathrm{T}}(\mathrm{mL} / 100 \mathrm{~g})$ & $0.78 \pm 0.05$ & $0.79 \pm 0.03$ & $0.78 \pm 0.03$ \\
\hline $\mathrm{V}_{\mathrm{T}} / \mathrm{T}_{\mathrm{I}}(\mathrm{mL} / 100 \mathrm{~g} / \mathrm{s})$ & $4.09 \pm 0.29$ & $3.74 \pm 0.27$ & $4.89 \pm 0.30 * \dagger$ \\
\hline
\end{tabular}

Baseline values were measured just before exposure to acute hypoxia on $\mathrm{p} 35$ or $\mathrm{p} 47$. All values are mean \pm SEM. Abbreviations: TR, rectal temperature; $\dot{\mathrm{V}}$, minute ventilation; $\mathrm{fR}$, respiratory frequency; VT, tidal volume; VT/TI, respiratory drive. P21N = weaned on p21and raised in normoxia; P15N = weaned on 15 and raised in normoxia; $\mathrm{P} 15 \mathrm{RH}=$ weaned on 15 and exposed to recurrent hypoxia.

$*, \uparrow$ Differences between P15RH and P15N, and between P15RH and P21N, respectively. On both p35 and p47, minute ventilation, respiratory frequency, and respiratory drive after recurrent hypoxia exceeded those in the early weaned control group. Furthermore, minute ventilation on p 35 and respiratory drive on p47 also exceeded those in the normally weaned control groups. 

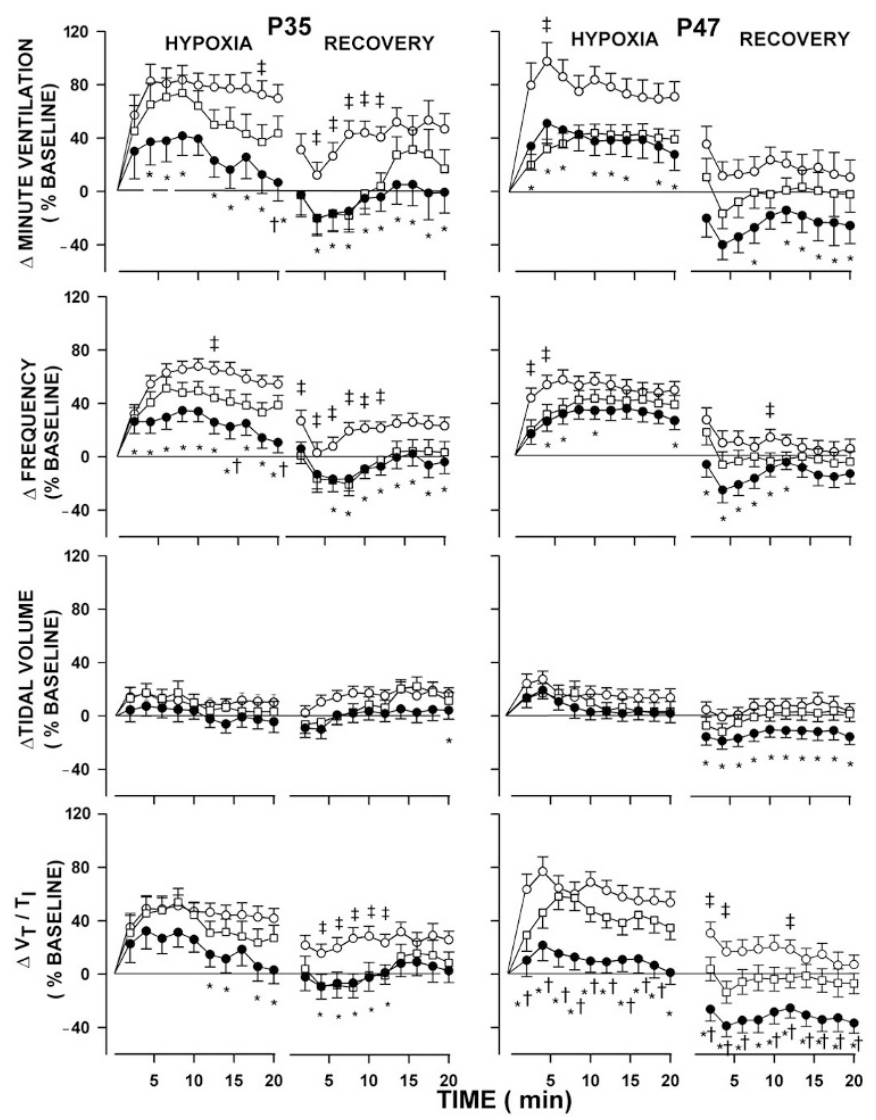

Figure 1. Minute ventilation, respiratory frequency, tidal volume, and $V_{T} / T_{I}$ (tidal volume/inspiratory time, representing respiratory drive) during exposure to acute hypoxia and during recovery from the acute hypoxia in normoxia on p35 and p47 in male rats. All values are calculated as percentage change from baseline and expressed as mean \pm SEM. Rat groups comprised pups weaned on p15 and raised in normoxia $(\bigcirc, \mathrm{P} 15 \mathrm{~N} ; n=11$ on $\mathrm{p} 35, n=9$ on $\mathrm{p} 47)$, weaned on $\mathrm{p} 21$ and raised in normoxia $(\square, \mathrm{P} 21 \mathrm{~N} ; n=7$ on $\mathrm{p} 35, n=$ 8 on $\mathrm{p} 47$ ), or weaned on p15 and exposed to recurrent hypoxia on p17-p33 (๑, P15RH; $n=6$ on p35, $n=8$ on p47). *Denotes a difference between $\mathrm{P} 15 \mathrm{RH}$ and P15N; †denotes a difference between P15RH and P21N; and $\ddagger$ denotes a difference between $\mathrm{P} 15 \mathrm{~N}$ and $\mathrm{P} 21 \mathrm{~N}$. Overall, a prior exposure to recurrent hypoxia resulted in a lesser respiratory responsiveness compared with control both during an acute hypoxia and during the recovery from an acute hypoxia on both p35 and p47. For further details and statistics, please see the text.

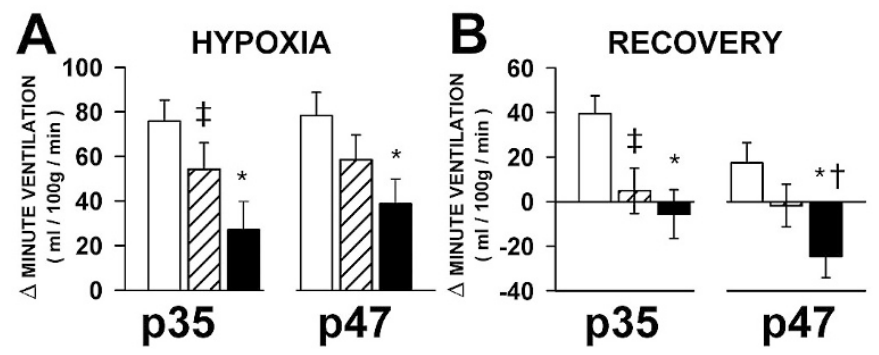

Figure 2. Average minute ventilation change over the entire period of acute hypoxia $(A)$ or of recovery from acute hypoxia $(B)$ in male rats on p35 and p47. Overall, the average minute ventilation response during and after acute hypoxia was smaller in groups exposed to recurrent hypoxia than in control

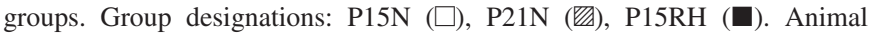
numbers and symbols are identical to those in Figure 1. For further details, please see the text.
$\mathrm{F}(9,387)=2.525, p=0.008$; tidal volume: $\mathrm{F}(9,387)=$ $10.211, p<0.0001$ and respiratory drive: $\mathrm{F}(9,387)=2.177$, $p=0.0228)$. Differences among groups were found, however, in minute ventilation $(\mathrm{F}(2,43)=11.201, p=0.0001)$, respiratory frequency $(\mathrm{F}(2,43)=6.606, p<0.0001)$, tidal volume $(\mathrm{F}(2,43)=5.529, p=0.0073)$ and respiratory drive $(\mathrm{F}(2,43)$ $=16.91, p<0.0001)$.

On $\mathrm{p} 35$ and $\mathrm{p} 47, \mathrm{P} 15 \mathrm{RH}$ rats displayed a greater percentage reduction in minute ventilation than did $\mathrm{P} 15 \mathrm{~N}$ controls $(p=$ 0.0001), lasting through most of the recovery period (pairwise comparisons, Fig. 1). On both test days, P15RH rats also displayed a greater reduction in raw average minute ventilation responses during recovery than did $\mathrm{P} 15 \mathrm{~N}$ or $\mathrm{P} 21 \mathrm{~N}$ rats $(\mathrm{F}(2,43)=11.201, p=0.0001$, Fig. $2 B)$. On p35, minute ventilation, both as percentage change and as average response, was higher in $\mathrm{P} 15 \mathrm{~N}$ than in $\mathrm{P} 21 \mathrm{~N}$ rats $(p=0.005$; pairwise comparisons, Fig. 1; Fig. $2 B$, respectively).

Respiratory frequency displayed greater reduction in $\mathrm{P} 15 \mathrm{RH}$ rats than in $\mathrm{P} 15 \mathrm{~N}$ rats over most of the recovery period on p35 and p47 ( $p=0.0001$; pairwise comparisons, Fig. 1), with additional differences between P15N and P21N groups $(p=0.003$; pairwise comparisons, Fig. 1). Average inspiratory time response on $\mathrm{p} 47$ decreased to a lesser extent in $\mathrm{P} 15 \mathrm{RH}$ than in $\mathrm{P} 15 \mathrm{~N}$ or $\mathrm{P} 21 \mathrm{~N}$ rats $(p<0.0001, p=0.0056$, respectively), whereas average expiratory time response decreased to the same extent in all groups, mirroring the changes in respiratory frequency (data not shown).

Tidal volume during recovery was reduced to a greater extent in P15RH rats than in $\mathrm{P} 15 \mathrm{~N}$ or $\mathrm{P} 21 \mathrm{~N}$ rats on both test days ( $p=0.0013$ and $p=0.0419$, respectively), and this reduction was more readily seen throughout the recovery period on p47 (pairwise comparisons, Fig. 1).

On both p35 and p47, the reduction in respiratory drive was greater in the $\mathrm{P} 15 \mathrm{RH}$ groups than in the $\mathrm{P} 15 \mathrm{~N}$ or the $\mathrm{P} 21 \mathrm{~N}$ groups ( $p<0.0001$ and $p=0.011$, respectively; Fig. 1). Moreover, whereas, on p35, both the P15RH and the P21N groups displayed a lower respiratory drive than the P15N group during the initial half of the recovery period, on $\mathrm{p} 47$, the respiratory drive of the $\mathrm{P} 15 \mathrm{RH}$ group was lower than that of the two control groups and remained below baseline levels throughout the recovery period (test day interaction, $\mathrm{F}(9,387)$ $=2.52, p=0.0081$ ) (Fig. 1). On both $\mathrm{p} 35$ and p47, the respiratory drive response of the $\mathrm{P} 15 \mathrm{~N}$ group exceeded that of the P21N group ( $p=0.0015$; Fig. 1$)$.

On p35, P15RH rats took 8 min, whereas P15N and P21N animals took $<2$ and $4 \mathrm{~min}$, respectively, to reach prehypoxic baseline minute ventilation. Similarly, on p47, P15RH animals required $10 \mathrm{~min}$ to reach baseline minute ventilation compared with $<2$ min for the P15N or P21N groups. Neither tidal volume nor respiratory drive of the P15RH rats returned to baseline over the 20 min of measurement on p47 (Fig. 1). In summary, prior exposure to recurrent hypoxia resulted in reduced respiratory responses during a subsequent acute hypoxia, followed by a more profound and longer respiratory depression during the posthypoxic recovery period.

Effects of sex and weaning on respiratory indices and growth. On p35, there were no differences between males and females, or any interactions between sex and study group, time in 
hypoxia and post hypoxia, or test day in minute ventilation, respiratory frequency, tidal volume, or respiratory drive.

There were no differences in weight between the sexes on the day of weaning (Fig. 3). Body weight increased from p15 to $\mathrm{p} 35(\mathrm{~F}(9,531)=1074.05, p<0.0001)$ in a manner dependent on sex and time of weaning: Overall, male rats weighed more than female rats regardless of weaning history $(\mathrm{F}(1,59)=8.60, p=0.0048)$. Due to a weight loss of $12 \%$ in the pups weaned on p15 24-48 h after weaning, a small weight difference between those and pups weaned on p 21 was detectable in both sexes until p30, but was no longer present on test day p35 (day by weaning interaction: $\mathrm{F}(18,531)=$ $4.285, p<0.0001)$. In addition, the small weight difference noted in females between the P15RH group and the P15N or P21N group was no longer seen on p35 (Fig. 3).

\section{DISCUSSION}

This study shows that, relative to early weaned controls, rats that had been exposed to recurrent daily hypoxia from $\mathrm{p} 17$ through p33 displayed reduced responses in minute ventilation, respiratory frequency, and respiratory drive during subsequent acute hypoxia on both p35 and p47. For minute ventilation and respiratory frequency on both test days, and for respiratory drive on $\mathrm{p} 47$, this reduction occurred early in the course of the acute hypoxia and lasted throughout much of the hypoxic exposure. Such reduction in minute ventilation, primarily driven by the changes in respiratory timing, can therefore be defined as an attenuation in respiratory responsiveness to acute hypoxia. This attenuation was seen not only as a percentage decrease from baseline but also as a significant decrease in average minute ventilation for the entire period of the acute hypoxia.

None of the four respiratory parameters displayed a difference in responsiveness to hypoxia between the two test days. It can thus be concluded that the magnitude of respiratory attenuation of the hypoxic response persisted for at least $14 \mathrm{~d}$ after the cessation of the recurrent hypoxia, a relatively long period in a rodent's life span.
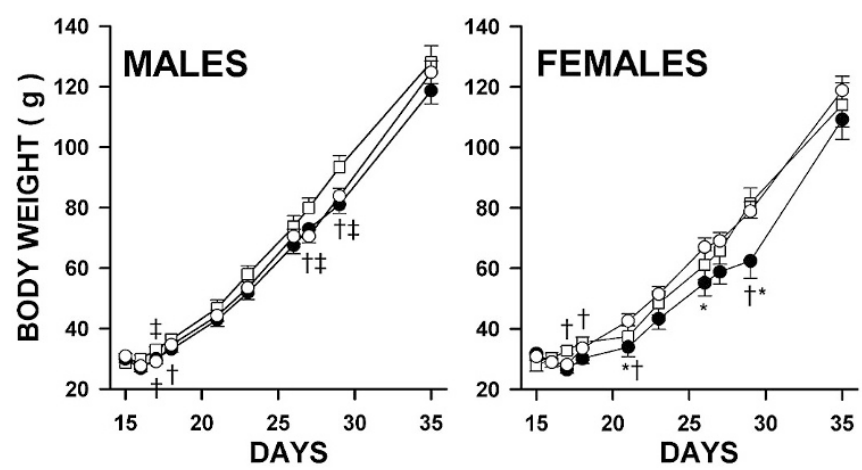

Figure 3. Body weight (g, mean \pm SEM) of rat pups $(n=65$ in total) from p15 through p35 that were either weaned on p15 and exposed to normoxia $(\bigcirc$, P15N; 19 males, 12 females), weaned on p21 and exposed to normoxia ( $\square$, P21N; 9 males, 5 females), or weaned on p15 and exposed to recurrent hypoxia from p17-p33 (, P15RH; 14 males, 6 females). *Denotes a difference between P15RH and P15N; †denotes a difference between P15RH and $\mathrm{P} 21 \mathrm{~N}$; and $\ddagger$ denotes a difference between P15N and P21N. Overall, males weighed more than females. After a transient weight loss after early weaning, all rats gained weight and displayed no effect of weaning or hypoxia on testing day p35. For details and statistics, please see the text.
In addition to the attenuation during acute hypoxia, prior recurrent hypoxia suppressed the recovery of respiration from acute hypoxia: Minute ventilation and respiratory frequency in the P15RH group were lower than those in the P15N group and displayed a longer delay in returning to baseline values. Moreover, tidal volume and respiratory drive in the $\mathrm{P} 15 \mathrm{RH}$ group on p47 did not return to baseline over the entire $20 \mathrm{~min}$ of measurement after acute hypoxia. The large attenuation in respiratory drive both during and after the acute hypoxia in the $\mathrm{P} 15 \mathrm{RH}$ group on $\mathrm{p} 47$ was partly due to the relatively unaltered inspiratory time in that group on that test day. The findings of greater suppression in tidal volume and respiratory drive during posthypoxic recovery on $\mathrm{p} 47$ than on $\mathrm{p} 35$ suggest that respiratory attenuation after prior recurrent hypoxia not only persists long term but may even increment with age. The attenuation of respiration both during and after acute hypoxia following recurrent hypoxia may represent a dysfunctional adaptation in respiratory control.

Because we have not studied longer postnatal intervals after the recurrent hypoxia, we cannot predict whether such respiratory attenuation is permanent or not. The studies of Bavis et al. (10) and Okubo and Mortola $(11,12)$, in which neonatal rat pups were exposed to continuous hypoxia with their dams and tested 7-8 and $12 \mathrm{wk}$ later, respectively, provide evidence that diminished ventilatory responses after such continuous hypoxia last into rat adulthood.

The present findings must be specific to developmental processes, as long-term intermittent and/or continuous hypoxia in adult (22) or p37 rats (12) did not attenuate responses to subsequent immediate or later acute hypoxia. Indeed, after intermittent or continuous hypoxia imposed during adulthood (22), ventilatory responses to an immediate subsequent acute hypoxia were enhanced. Those adult rats (22) displayed a transient sharp rise in minute ventilation during the posthypoxic recovery, unlike the attenuation after recurrent hypoxia seen in our developmental study. Whereas these discrepant findings may be related to differences in the patterns of the chronic hypoxia, they also point to the desirability of matching the age range of the model to the clinical condition being simulated.

The clinical studies that demonstrated lingering effects of prior hypoxia, albeit on neurocognitive functions, involved intermittent recurrent hypoxia with the associated sleep disturbances in children (4,7-9). In contrast, neither the present study, nor those by Bavis et al. (10) and Okubo and Mortola $(11,12)$ used an intermittent hypoxic pattern. Direct comparison of responses to acute intermittent versus continuous hypoxic challenge in piglets showed that the intermittent pattern produced greater decline in respiratory indices (20). In addition, gestational intermittent hypoxia in rats resulted in a decrease of ventilatory responses to subsequent acute hypoxia on p5 (21). One might therefore expect that intermittent recurrent hypoxia in rats throughout the developmental age used in this study would further enhance the decline in ventilatory responses to subsequent acute hypoxia in later life.

The attenuation of the respiratory functions occurred not only during the initial period of rapid peripheral chemosensory responses but persisted throughout the hypoxic challenge. Therefore, this attenuation likely involved central effects of 
hypoxia. Possible mechanisms might include adaptations in fundamental respiratory-related neurophysiological processes, integrative brainstem functions, and receptor/ligand/ transporter systems, and/or more permanent changes, including neuronal damage and death (e.g. 23-28). Assuming that $\mathrm{V}_{\mathrm{T}} / \mathrm{T}_{\mathrm{I}}$ provides a reliable index of respiratory drive in the intact animal, the sizable attenuation of respiratory drive during and after hypoxia in $\mathrm{P} 15 \mathrm{RH}$ versus the $\mathrm{P} 15 \mathrm{~N}$ and $\mathrm{P} 21 \mathrm{~N}$ rats on p47 as well as the suppression below baseline of both respiratory drive and tidal volume during the recovery from acute hypoxia on $\mathrm{p} 47$ suggest a possible involvement of the respiratory central pattern generator in producing this attenuation (29).

The lack of any observed differences in responsiveness during and after acute hypoxia between males and females stands in contrast to the presence of such differences in a previous report (18). The discrepancy in the findings can perhaps be attributed to a difference in the postnatal age range of hypoxic exposure and/or in the modality of maternal separation. Unlike the experimental design in the previous study (18) that comprised separating and isolating pups from their dams and litter-mates for several hours per day and exposing them to hypoxia during the p3-p14 "stress hyporesponsive period," the pups in the present study were weaned at a developmental stage that is beyond that vulnerable period, characterized by an immaturity in the hypothalamic-pituitaryadrenal axis $(15,16)$. Tactile and chemical stimuli from the mother are necessary for the maturation of this axis at that time, and maternal separation and pup isolation for as little as $3 \mathrm{~h}$ per day during that time window can induce permanent abnormalities in emotional, behavioral and neuroendocrine stress responses $(15,16,30)$. To further minimize the effects of maternal separation, the present study included preweaning handling, nutritional supplement, group housing, and continued handling after weaning. Nevertheless, our findings of small, albeit transient, body weight differences between early and normally weaned pups, of inconsistent differences between the P15RH and the P21N rats, and of occasional differences between the P15N and the P21N control groups indicate that effects of early weaning were still present. When there were differences between the two control groups, the $\mathrm{P} 15 \mathrm{~N}$ rats consistently displayed larger respiratory responses than the P21N rats. Such heightened responses perhaps indicate the presence of maternal separation-related potentiation of respiratory responses to hypoxia in male rats (18).

In summary, male rat pups exposed to recurrent hypoxia for 17 consecutive days and tested for respiratory responsiveness to acute hypoxia 2 and $14 \mathrm{~d}$ later displayed an attenuation of minute ventilation, respiratory frequency and respiratory drive during and following the hypoxia, and of tidal volume during the recovery from hypoxia. The magnitude of the attenuation during acute hypoxia did not differ between the two test days. During posthypoxic recovery, the attenuation of respiratory drive increased from the first to the second test day. These results indicate the presence of persistent, relatively long-term abnormalities in respiratory control following recurrent hypoxia during postnatal development, and suggest that such hypoxia in humans might reduce the ability to respond adequately to subsequent hypoxic events in adulthood.

\section{REFERENCES}

1. Bass JL, Corwin M, Gozal D, Moore C, Nishida H, Parker S, Schonwald A, Wilker RE, Stehle S, Kinane TB 2004 The effect of chronic or intermittent hypoxia on cognition in childhood: a review of the evidence. Pediatrics 114:805-816

2. Nixon GM, Kermack AS, Davis GM, Manoukian JJ, Brown KA, Brouillette RT 2004 Planning adenotonsillectomy in children with obstructive sleep apnea: the role of overnight oximetry. Pediatrics 113:e19-e25

3. Marcus CL, Lutz J, Carroll JL, Bamford O 1998 Arousal and ventilatory responses during sleep in children with obstructive sleep apnea. J Appl Physiol 84:1926-1936

4. Schechter MS 2002 Technical report: diagnosis and management of childhood obstructive sleep apnea syndrome. Pediatrics 109:e69 (1-20)

5. Lipton AJ, Gozal D 2003 Treatment of obstructive sleep apnea in children: do we really know how? Sleep Med Rev 7:61-80

6. O'Brien LM, Mervis CB, Holbrook CR, Bruner JL, Smith NH, McNally N, McClimment MC, Gozal D 2004 Neurobehavioral correlates of sleep-disordered breathing in children. J Sleep Res 13:165-172

7. Ali NJ, Piston D, Scrawling JR 1996 Sleep disordered breathing: effects of adenotonsillectomy on behaviour and psychological functioning. Eur J Pediatr 155:56-62

8. Gozal D 1998 Sleep-disordered breathing and school performance in children. Pediatrics 102:616-620

9. Harvey JM, O'Callaghan MJ, Wales PD, Harris MA, Masters JB 1999 Six-month follow-up of children with apnoea. J Paediatr Child Health 35:136-139

10. Bavis RW, Olson EB, Vidruk EH, Fuller DD, Mitchell GS 2004 Developmental plasticity of the hypoxic ventilatory response in rats induced by neonatal hypoxia. J Physiol 557:645-660

11. Okubo S, Mortola JP 1988 Long-term respiratory effects of neonatal hypoxia in the rat, J Appl Physiol 64:952-958

12. Okubo S, Mortola JP 1990 Control of ventilation in adult rats hypoxic in the neonatal period, Am J Physiol 259:R836-R851

13. Dobbing J, Sands J 1979 Comparative aspects of brain growth spurt. Early Hum Dev 3:79-83

14. Brown KA, Laferrière A, Moss IR 2004 Recurrent hypoxemia in young children with obstructive sleep apnea is associated with reduced opioid requirement for analgesia. Anesthesiology 100:806-810

15. Francis DD, Caldji C, Champagne F, Plotsky PM, Meaney MJ 1999 The role of corticotropin releasing factor norepinephrine systems in mediating the effects of early experience on the development of behavioral and endocrine responses to stress. Biol Psychiatry 46:1153-1166

16. Brake WG, Zhang TY, Diorio J, Meaney MJ, Gratton A 2004 Influence of early postnatal rearing conditions on mesocorticolimbic dopamine and behavioural responses to psychostimulants and stressors in adult rats. Eur J Neurosci 19:18631874

17. Simakajornboon N, Vlasic V, Li H, Sawnani H 2004 Effect of prenatal nicotine exposure on biphasic hypoxic ventilatory response and protein kinase $\mathrm{C}$ expression in caudal brainstem of developing rats. J Appl Physiol 96:2213-2219

18. Genest SE, Gulemetova R, Laforest S, Drolet G, Kinkead R 2003 Neonatal maternal separation and sex-specific plasticity of the hypoxic ventilatory response in awake rat. J Physiol 554:543-557

19. Mortola JP, Frappell PB 1998 On the barometric method for measurements of ventilation, and its use in small animals. Can J Physiol Pharmacol 76:937-944

20. Waters KA, Beardsmore C, Paquette J, Meehan B, Côté A, Moss IR 1996 Respiratory responses to rapid-onset, repetitive vs continuous hypoxia in piglets. Respir Physiol 105:135-142

21. Gozal D, Reeves SR, Row BW, Neville JJ, Guo SZ, Lipton AJ 2003 Respiratory effects of gestational intermittent hypoxia in the developing rat. Am J Respir Crit Care Med 167:1540-1547

22. Reeves SR, Gozal E, Guo SZ, Sachleben LR, Jr., Brittian KR, Lipton AJ, Gozal D 2003 Effect of long-term intermittent and sustained hypoxia on hypoxic ventilatory and metabolic responses in the adult rat. J Appl Physiol 95:1767-1774

23. Moss IR 2005 Neural control of breathing. In: Hamid Q, Shannon J, Martin J, (eds) Physiologic Basis of Respiratory Disease. BC Decker, Hamilton, ON, Canada, pp 251-261

24. Carroll JL 2003 Plasticity in respiratory motor control: invited review: developmental plasticity in respiratory control. J Appl Physiol 94:375-389

25. Macey PM, Henderson LA, Macey KE, Alger JR, Frysinger RC, Woo MA, Harper RK, Yan-Go FL, Harper RM 2002 Brain morphology associated with obstructive sleep apnea. Amer J Respir Crit Care Med 166:1382-1387

26. Gozal D, Row BW, Kheirandish L, Liu R, Guo SZ, Qiang F, Brittain KR 2003 Increased susceptibility to intermittent hypoxia in aging rats: changes in proteosomal activity, neuronal apoptosis and spatial function. J Neurochem 86:1545-1552

27. Gozal E, Row BW, Shurr A, Gozal D 2001 Developmental differences in cortical and hippocampal vulnerability to intermittent hypoxia in the rat. Neurosci Lett 305:197-201

28. Payne RS, Goldbart A, Gozal D, Schurr A 2004 Effect of intermittent hypoxia on long-term potentiation in rat hippocampal slices. Brain Research 1029:195-199

29. Ramirez J-M, Viemari J-C 2005 Determinants of inspiratory activity. Respir Physiol Neurobiol 147:145-157

30. Wigger A, Neumann ID 1998 Periodic maternal deprivation induces genderdependent alterations in behavioural and neuroendocrine responses to emotional stress in adult rats. Physiol Behav 66:293-302 